\title{
Electronic structure of disordered Fe-V alloys
}

\author{
J. C. Krause \\ Instituto de Física, Universidade Federal de Rio Grande do Sul, UFRGS, Porto Alegre CEP 91501-970, RGS, Brazil \\ C. Paduani \\ Departamento de Física, Universidade Federal de Santa Catarina, UFSC, Florianópolis CEP 88040-900, SC, Brazil \\ J. Schaff and M. I. da Costa, Jr. \\ Instituto de Física, Universidade Federal de Rio Grande do Sul, UFRGS, Porto Alegre CEP 91501-970, RGS, Brazil
}

(Received 26 June 1997; revised manuscript received 11 September 1997)

\begin{abstract}
The first-principles discrete variational method is employed to investigate the electronic structure and local magnetic properties of disordered Fe-V alloys. The spin-polarized case is considered in the formalism of the local-spin-density approximation, with the exchange-correlation term of von Barth-Hedin. The effect on the local magnetic properties of adding $\mathrm{V}$ atoms in the immediate neighborhood of iron atoms is investigated. The partial density of states, hyperfine field $\left(H_{c}\right)$, magnetic moment $(\mu)$, and isomer shift are obtained for the central atom of the cluster. For the impurity $\mathrm{V}$ atom in the bcc iron host the calculated values for $H_{c}$ and $\mu$ are $-203 \mathrm{kG}$ and $-0.86 \mu_{B}$, respectively. The isolated $\mathrm{Fe}$ atom in a bcc vanadium host exhibits a collapsed moment and acts as a receptor for electrons. In ordered alloys the calculations indicate also a vanishing moment at iron sites. [S0163-1829(98)05901-3]
\end{abstract}

\section{INTRODUCTION}

The Fe-V alloy system is formed by a bcc solid solution at high temperatures. ${ }^{1}$ At the equiatomic composition is formed an ordered intermetallic compound at low temperatures. This phase diagram has been intensively studied in the last decades, mostly concerning the order-disorder transitions. Recently, the low-temperature topology of the metastable phase diagram was established for this system. ${ }^{2}$ The critical temperatures for the order-disorder transformation was measured by Seki et al. as a function of the concentration. $^{3}$ The results showed a nearly symmetric parabolic behavior for this dependence. Ryzhenko et al. have investigated the local magnetic characteristics of disordered $\mathrm{Fe}-\mathrm{V}$ alloys on the basis of an analysis of the functions of the densities of the distribution of the magnetic moments from experimental results. ${ }^{4}$ They obtained a very wide distribution of the magnetic moments of the iron atoms, corresponding to a strong influence of the local atomic environment around them.

In amorphous alloys the appearance of local moments are due to some degree of atomic ordering. In crystalline systems, it is also disputed whether this same process could occur. Moreover, some kind of dependence of the magnetic properties on the degree of atomic ordering is expected. Experimental results have indicated that a considerable average moment reduction occurs when the disordered bcc alloys are transformed into an ordered phase by low temperature annealing of Fe-V disordered alloys. ${ }^{5}$ Magnetic crystalline alloys which exhibit a variable degree of atomic ordering are not usual. Owing to the fact that for the Fe-V alloy system the disordered phase it is obtained over the whole composition range, besides the fact that the region where atomic ordering into an intermetallic compound can take place is rather large, makes it a good candidate to investigate these trends.

Mössbauer spectroscopy results have indicated that the iron moment reduction is not an apparent effect associated with possible deviations from a ferromagnetic moment arrangement, and have presented evidence that the magnetic properties of iron-rich alloys depend strongly on the degreee of atomic ordering. ${ }^{5}$ Experimental results show that $\mathrm{Fe}-\mathrm{V}$ alloys are ferromagnetic up to 75 at. $\% \mathrm{~V}$, and that the vanadium atoms exhibit a small magnetic moment of $-0.9 \mu_{B}$ as impurity in a bcc iron host, antiferromagnetically coupled with the iron moments. ${ }^{6-9}$

The dependence of the local moments with the composition in Fe-V alloys was investigated by Maksymowicz by means of a Green function with the continued-fraction technique, including short-range atomic ordering as well as atomic long-range order in a two-sublattice model. ${ }^{10} \mathrm{~A}$ linear decrease of the magnetic moment with the increase of $\mathrm{V}$ atoms in the vicinity of iron atoms was obtained from the calculations. An antiferromagnetic coupling between Fe and $\mathrm{V}$ atoms was obtained, with a value of $-1.7 \mu_{B}$ for the $\mathrm{V}$ moment in a bcc iron host. The results indicate also that there is no minimum number of $\mathrm{Fe}$ atoms required in the vicinity of a $\mathrm{Fe}$ atom in order for there to appear a local moment therein, and besides, that no magnetic moments are expected on the $\mathrm{V}$ atoms in the ordered phases. However, the lack of experimental data do not permit us to confirm that only iron atoms contribute to the bulk magnetization in the ordered state. Furthermore, even for an iron atom surrounded by a neighborhood fulfilled with $\mathrm{V}$ atoms is observed the formation of a local moment. The rate of decrease of the iron magnetic moment with the introduction of $\mathrm{V}$ atoms in its immediate neighborhood was obtained as $0.21 \mu_{B}$ per atom from the calculations. The results of Hamada and Miwa give $0.18 \mu_{B},{ }^{11}$ whereas the experimental result is $0.10 \mu_{B}{ }^{8}$ 
The electronic structure of $\mathrm{Fe}-\mathrm{V}$ superstructures have been calculated with the discrete variational (DV)- $X_{\alpha}$ method. ${ }^{12}$ The results have indicated that the magnetic moment at iron atoms does not decrease with increasing the number of vanadium atoms in its vicinity, as it does for $H_{c}$. Besides, a linear correlation was obtained between $H_{c}$ and the isomer shift (IS).

In this paper we investigate the electronic structure of 15-atom clusters representing bcc iron and Fe-V alloys. The DV method ${ }^{13}$ is employed with the exchange-correlation term of von Barth-Hedin, ${ }^{14}$ in the formalism of the localspin-density (LSD) approximation. In the next section we present some details of the calculational procedure, and in the remaining sections we discuss our results.

\section{PROCEDURES OF CALCULATION}

The DV method uses the Hartree-Fock-Slater model and the linear combination of atomic orbitals (LCAO) to build up the molecular orbitals. A minimal basis was adopted, which includes $1 s-4 p$ orbitals for both iron and vanadium. The numerical atomic orbitals are generated by solving the atomic problem, in the $X_{\alpha}$ approximation. A spherically averaged potential is used around each atom, in order to obtain more contracted valence orbitals. Potential wells located at outer atoms in the crystal region were truncated at the Fermi energy, to prevent the migration of electrons from the clusters towards low-lying states located on crystal atoms. The molecular clusters were built up with the experimental value of 5.41606 a.u. for the lattice spacing of bcc iron. The origin of coordinates is at the body-centered site. The microcrystal was extended up to 20 a.u., including few hundred of atoms. This embedding scheme is well described elsewhere in the literature. ${ }^{15}$ The electronic Hamiltonian for a spin $\sigma$ can be written as

$$
H_{\sigma}=-\frac{1}{2} \nabla^{2}+V_{c}(\rho)+V_{\mathrm{xc}}\left(\rho_{\sigma}\right)
$$

and the total electronic charge density is

$$
\rho(\vec{r})=\sum_{j} n_{j \sigma}\left|\psi_{j \sigma}(\vec{r})\right|^{2},
$$

where $n_{j \sigma}$ is the molecular orbital population. The Coulombian potential $V_{c}$ includes both electronic and nuclear parts, and $V_{\text {xc }}$ is the exchange-correlation.

The one-electron equation

$$
\left(H_{\sigma}-\epsilon_{J \sigma}\right) \psi_{j \sigma}(\vec{r})=0,
$$

is solved self-consistently in the LCAO approximation allowing for different spacial parts of the molecular orbitals for different spin. The matrix elements of the Hamiltonian and overlap are evaluated by direct numerical integration procedures, using a random sampling method, where a sum of Fermi-type functions around each atom is adopted as the distribution function of the sample points. ${ }^{16}$

The central atom of each cluster is the probe atom, which has bulklike properties. The truncation of the cluster does not allow sufficient wave-function delocalization to take place, and so, the local magnetic moments tend to be more atomiclike. Thus, a finite cluster effect is, departing from the central atom toward the surface of the cluster, one observes increasing values for the magnetic moments. Actually, only the central atom of each cluster, which is in the origin of the coordinate system, has the closest environment to the crystalline ambient, and is the more adequate probe to give reliable results for the bulk properties.

\section{Density of states, hyperfine fields, and isomer shifts}

The partial density of states (PDOS) for a particular level is constructed by weighing each molecular energy level by the corresponding population, and then broadening each level into a Lorentzian width of $0.085 \mathrm{eV}$, which is consistent with our precision, in order to obtain a continuous curve.

The nonzero spin density of electrons $s$ at the nucleus $\left[\Delta \rho_{S}(0)\right]$ gives rise to the Fermi contact hyperfine field which can be obtained by

$$
H_{c}=524.3 \times \Delta \rho_{S}(0) \mathrm{kG} .
$$

The core electrons contribution is obtained by the selfconsistent atomic calculations in the $X_{\alpha}$ approximation with the exchange coefficient of 0.667 for the deep $1 s, 2 s$, and $3 s$ core orbitals. This is obtained by using the converged electronic configurations from the molecular calculations, after an appropriate diagonal weighed population analysis to obtain the atomic-orbital populations. The conduction-electron contribution is obtained straight from the molecular orbitals. This approximation is advisable since there is not sufficient variational freedom with the minimal basis used here to get reliable numerical accuracy to the contribution of the core levels.

The isomer shift (IS) value is obtained directly from

$$
I S=-0.25 \times \Delta \rho_{C}(0) / \mathrm{mm} / \mathrm{s} \quad a_{0}^{3},
$$

where $\Delta \rho_{C}(0)$ is the total charge density difference at nucleus for absorber and source, respectively, relative to $\alpha$-iron. We excluded in this calculation the $1 s$ and $2 s$ contributions, considering the lack of precision for them. However, it is well known that the deep core electron orbitals give negligible contributions to the IS for iron atoms in different oxidation states.

\section{RESULTS AND DISCUSSION}

The partial density of states (PDOS) for the central iron atom is shown in Figs. 1(a)-1(c). The $3 d$ PDOS in Fig. 1(a) show a very pronounced peak for spin-up states for the $d$-electron density just below the Fermi surface. An intensive feature for $3 d$ hole states can be seen just above the Fermi energy $\left(E_{F}\right)$, in the spin-down band. In Figs. 1(b) and 1(c) one can verify the antiferromagnetic coupling between localized and conduction electrons. Figures 2(a) and 2(b) display the PDOS for the $\mathrm{V}$ atom isolated in the bcc iron matrix. Note how states tend to accumulate in the bottom of the $3 d$ band. In the virtual bound states there is a high peak very close to $E_{F}$ for spin-up states, whereas there still remain 

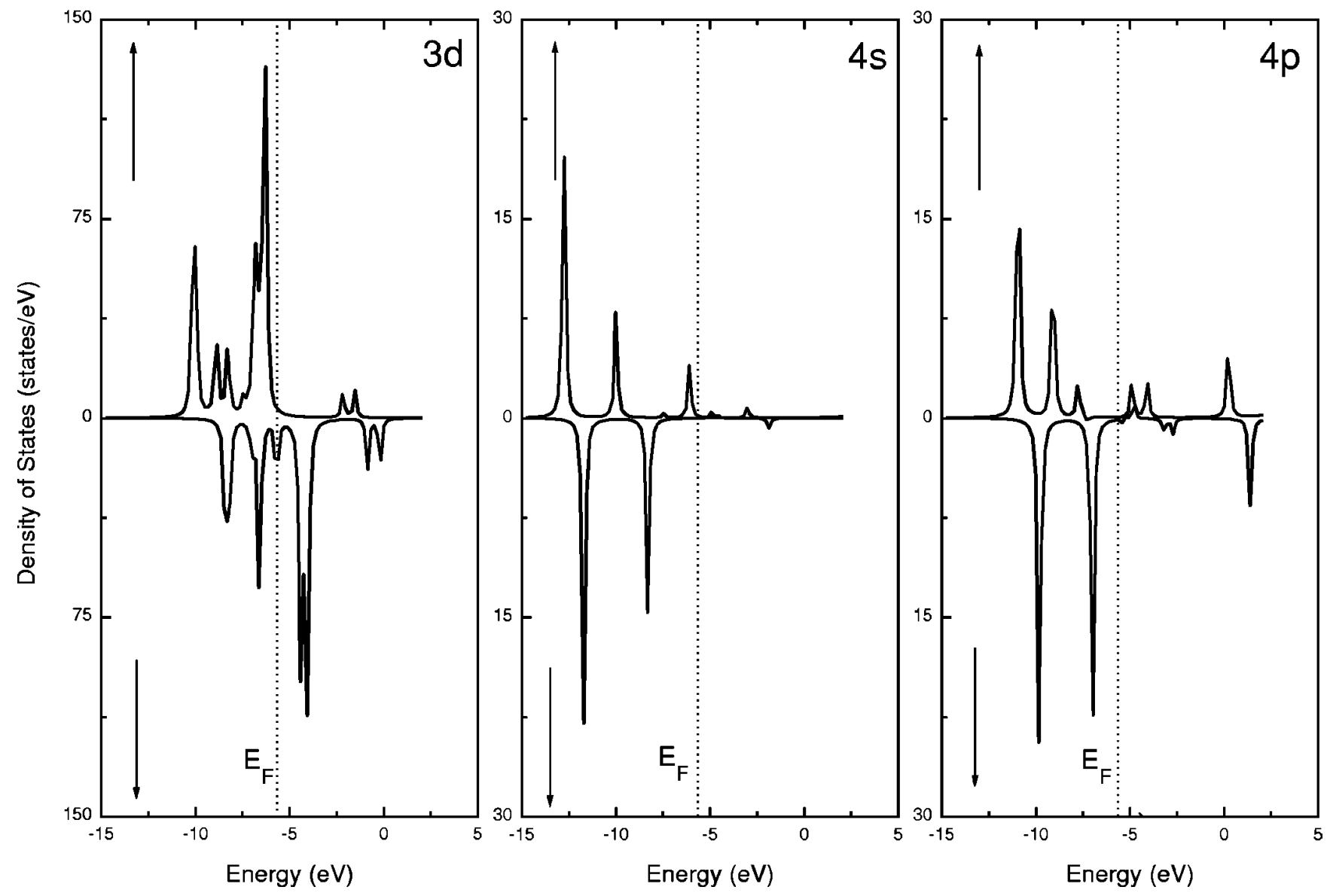

FIG. $1.3 d$ PDOS for pure bcc iron (a), $4 s$ (b), and $4 p$ (c).
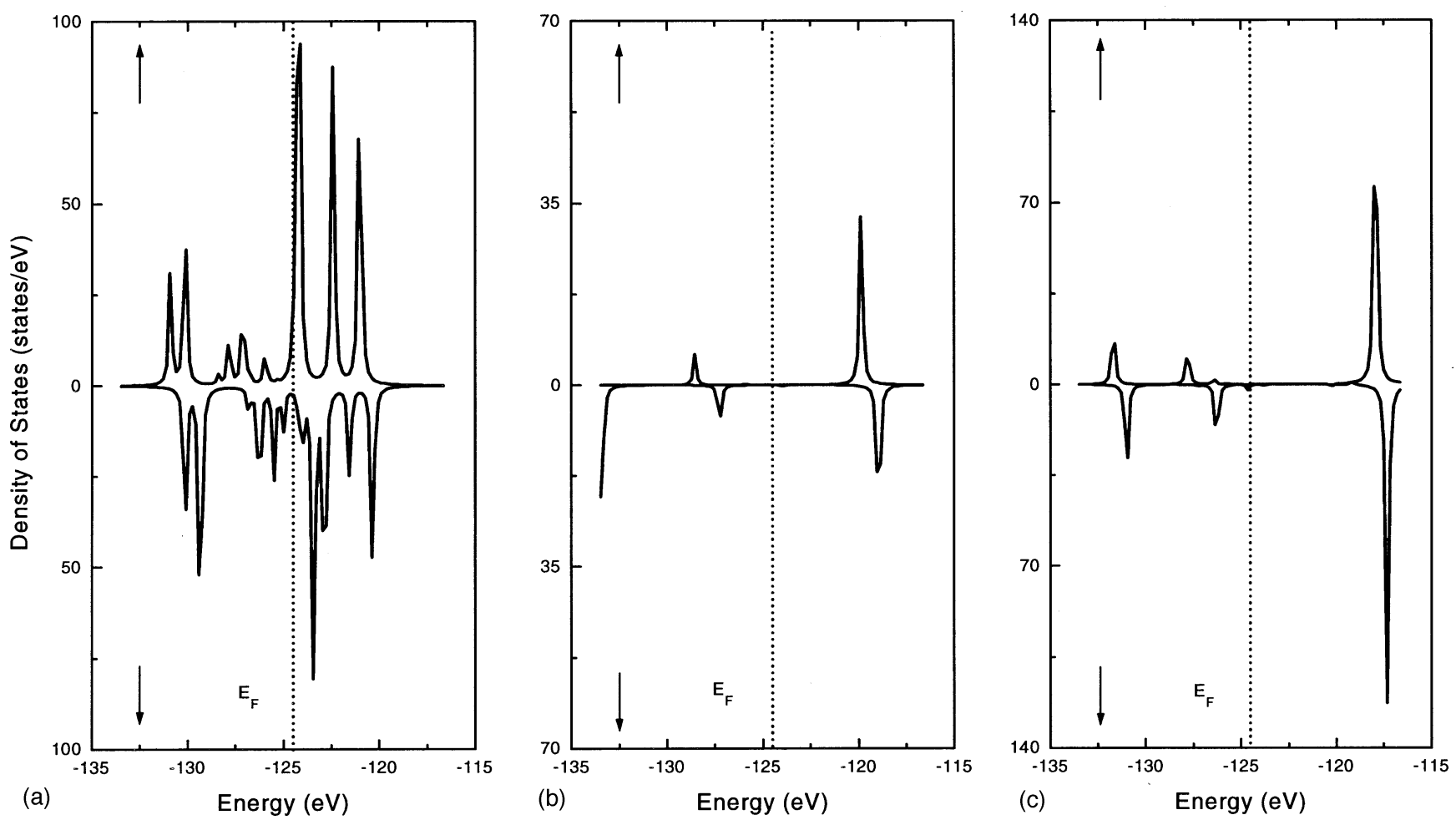

FIG. 2. $3 d$ PDOS for the central $\mathrm{V}$ atom (a), $4 s$ (b), and $4 p$ (c). 
TABLE I. Calculated results for the total magnetic moment $(\mu)$, the contribution from the conduction band $4 s+4 p\left(\mu_{c}\right)$, the hyperfine magnetic field $\left(H_{c}\right)$ and the isomer shift (IS), for the central atom of each cluster. $\mathrm{FeV}_{\mathrm{cr}}$ means one isolated $\mathrm{Fe}$ atom in vanadium matrix.

\begin{tabular}{lccccc}
\hline \hline Cluster & $\mu\left(\mu_{B}\right)$ & $\mu_{c}$ & $Q$ & $H_{c}(\mathrm{kG})$ & $\mathrm{IS}(\mathrm{mm} / \mathrm{s})$ \\
\hline $\mathrm{FeFe}_{8} \mathrm{Fe}_{6}$ & 2.29 & -0.35 & 0.17 & -347 & \\
$\mathrm{VFe}_{8} \mathrm{Fe}_{6}$ & -0.86 & -0.40 & -0.59 & -203 & \\
$\mathrm{FeVFe}_{7} \mathrm{Fe}_{6}$ & 1.93 & -0.22 & -0.47 & -178 & 0.07 \\
$\mathrm{FeV}_{2} \mathrm{Fe}_{6} \mathrm{Fe}_{6}{ }^{\mathrm{a}}$ & 1.83 & -0.10 & -0.72 & -101 & -0.10 \\
$\mathrm{FeV}_{2} \mathrm{Fe}_{6} \mathrm{Fe}_{6}{ }^{\mathrm{b}}$ & 1.40 & -0.08 & -0.77 & -59 & -0.07 \\
$\mathrm{FeV}_{4} \mathrm{Fe}_{4} \mathrm{Fe}_{6}{ }^{\mathrm{a}}$ & 1.83 & 0.00 & -0.73 & -110 & -0.17 \\
$\mathrm{FeV}_{4} \mathrm{Fe}_{4} \mathrm{Fe}_{6}{ }^{\mathrm{b}}$ & 1.50 & -0.05 & -0.76 & +41 & -0.07 \\
$\mathrm{FeV}_{8} \mathrm{Fe}_{6}$ & 0.61 & 0.03 & -0.54 & -104 & -0.25 \\
$\mathrm{FeFe}_{8} \mathrm{~V}_{6}$ & 0.31 & -0.02 & -0.52 & -41 & -0.06 \\
$\mathrm{FeV}_{8} \mathrm{~V}_{6}$ & 0.31 & 0.05 & 0.41 & +7 & -0.07 \\
$\mathrm{FeFe}_{8} \mathrm{VFe}_{5}$ & 1.56 & -0.24 & -0.74 & -190 & -0.05 \\
$\mathrm{FeV}_{\mathrm{cr}}$ & 0.06 & 0.01 & -1.10 & -18 & 0.04 \\
\hline \hline${ }^{\mathrm{a}} T_{d}$. & & & & & \\
${ }^{\mathrm{b}} C_{4 v}$. & & & &
\end{tabular}

empty states for spin-down electrons. The conduction band has a small occupation, with the states well localized at lower energies. Two pronounced peaks can be seen for hole states in Fig. 2(c). In Fig. 2(a) the Fermi energy falls into the minimum of the minority-spin density of states. The same result was obtained with multiple-scattering Korringa-KohnRostoker Green's-function tecnhique, as pointed out by Dritler et al. ${ }^{17}$ A continuous depolarization of the conduction band is observed, with the increase of the number of $\mathrm{V}$ atoms in the immediate vicinity of iron atoms in this host. Moreover, for dilute alloys the Stoner criterion is satisfied, where the rigid-band model can be applied and the ferromagnetic ordering is stabilized through the exchange interaction.

Table I presents the calculated results for the total magnetic moment $\mu$, alongside the contribution from conduction electrons $\mu_{c}(4 s+4 p)$, the ionization $Q$, the magnetic hyperfine field (Fermi contact term) $H_{c}$, and the isomer shift (IS), for the central atom of each cluster. The magnetic moment obtained for bcc iron is $2.29 \mu_{B}$, and for a V atom as an isolated impurity in this host it is $-0.86 \mu_{B}$. The experimental results are $2.217 \mu_{B}$ and $-0.9 \mu_{B}$, respectively. ${ }^{8}$ For the isolated $\mathrm{Fe}$ atom in bcc vanadium host our results indicate a null local moment and a receptor character for electrons. In the table one sees that the magnetic moment decreases with the increase of the number of vanadium atoms in the vicinity of iron sites. This behavior is confirmed from both theoretical and experimental results. $4,5,8,17$

The local symmetry plays a major rule, as can be checked out in Table I, for the clusters with 2 and $4 \mathrm{~V}$ nearestneighbor (NN) atoms. For the case of $1 \mathrm{Fe}$ atom surrounded by $14 \mathrm{~V}$ atoms, in the first and second neighborhood, our result is $0.31 \mu_{B}$ for the iron moment. Furthermore, for the cluster with $1 \mathrm{~V}$ next-nearest neighbor (NNN), we obtained $1.56 \mu_{B}$ for the central iron moment. In this case, the conduction-electron contribution to the local moment $\left(\mu_{c}\right)$ is $-0.24 \mu_{B}$. From the behavior of the $\mu_{c}$ value with dilution one should expect also a fast decrease of the critical temperature in these alloys, even in the ordered condition. Usually, the critical temperature decreases and the magnetization increases when the ordering process takes place. However, the experimental results have indicated in this case a reduction of both the magnetization as well as of the transition temperature in the ordering process. ${ }^{18}$

As can be seen in the table the contact field at iron sites decreases steadily with the addition of $\mathrm{V}$ atoms in this host, and depends strongly on the local symmetry on these sites. Actually, one feature depicted from these calculations is that an abrupt reduction in $H_{c}$ is expected when ordering takes place. The observed reduction in $H_{c}$ is due to the reduction of the contribution from deep-core electrons to the spin density at iron nuclei, an effect arising from the decrease of the polarization of the conduction band caused by the $3 d$ electrons which are going to occupy the spin-down band, which in turn decreases the local moment. The calculated results for $H_{c}$ at $0 \mathrm{~K}$ are $-347 \mathrm{kG}$, for pure bcc iron, and $-203 \mathrm{kG}$, for a $\mathrm{V}$ atom isolated in this matrix. From the calculations is estimated a reduction of about $170 \mathrm{kG}$ in $H_{c}$ at a iron site with the introduction of a $1 \mathrm{~V} \mathrm{NN}$ atom. With $1 \mathrm{NNN} \mathrm{V}$ atom this reduction is almost the same, which indicates a long-range mechanism in this case. From the table one sees also that no linear correlation exists between $H_{c}$ and the long-range order magnetization parameter. At the other hand, the IS value is quite sensitive to the occupancy of the neighboring shells, and depends strongly on the atomic ordering. The introduction of $\mathrm{V}$ atoms in the immediate vicinity of a $\mathrm{Fe}$ atom in this host causes an inflow of electrons into the iron sites, which grows up to $4 \mathrm{~V} \mathrm{NN}$ atoms, as can be observed for the $Q$ value in the table. Moreover, the magnetic moment of $\mathrm{V}$ atoms also decreases in this way towards the collapse with the onset of the atomic ordering ( $\mu=-0.038 \mu_{B}$ at $\mathrm{V}$ sites). This indicates that only the iron atoms contribute to the magnetization for maximum atomic long-range order.

\section{CONCLUSIONS}

In this work first-principles calculations are performed with the molecular cluster DV method in the LSD approximation to investigate the electronic structure and local magnetic properties of Fe-V alloys. The calculated $\mu$ value of an isolated $\mathrm{V}$ atom in the bcc iron matrix is in good agreement with the experimental result. The decrease of the local moment at iron atoms comes essentially from the reduction of the contribution from $3 d$ electrons. Our calculations indicate that in ordered alloys the iron atoms have a vanishing magnetic moment, in accord with experimental predictions. The local symmetry plays a major rule in these alloys with respect to $\mu, H_{c}$, and IS values. From these results one concludes that the appearance of local moments are strongly influenced by the atomic ordering in these alloys. The effect on the magnetization and $H_{c}$ behavior of the occupancy of the neighboring environment is expected to extend to at least a few shells of atoms. The introduction of $1 \mathrm{~V} \mathrm{NN}$ atom 
causes a reduction of about $170 \mathrm{kG}$ in the $H_{c}$ value and about $0.36 \mu_{B}$ in the local moment at iron sites. According to these results, the magnetic moment at both $\mathrm{Fe}$ and $\mathrm{V}$ atoms depends on the composition in this phase.

\section{ACKNOWLEDGMENTS}

This work was supported by $\mathrm{CNPq}$ and Finep, Brazilian agencies.
${ }^{1}$ M. Hansen and K. Ardenko, Constitution of Binary Alloys (McGraw-Hill, New York, 1958).

${ }^{2}$ J. M. Sanchez, M. C. Cadeville, V. Pierron-Bohnes, and G. Inden, Phys. Rev. B 54, 8958 (1996).

${ }^{3}$ J. J. Seki, M. Hagiwara, and T. Suzuki, J. Mater. Sci. 14, 2404 (1979).

${ }^{4}$ B. V. Ryzhenko and P. V. Gel'd, Sov. Phys. Solid State 29, 108 (1987).

${ }^{5}$ A. M. van der Kraan, D. B. de Mooij, and K. H. J. Buschow, Phys. Status Solidi A 88, 231 (1985).

${ }^{6}$ V. Jaccarino, L. R. Walker, and G. K. Wertheim, Phys. Rev. Lett. 14, 6 (1965).

${ }^{7}$ M. F. Collins and G. G. Low, Proc. Phys. Soc. London 86, 535 (1965).

${ }^{8}$ I. A. Campbell, Proc. Phys. Soc. London 89, 71 (1966).

${ }^{9}$ M. V. Nevitt and A. T. Aldred, J. Appl. Phys. 34, 463 (1963).

${ }^{10}$ A. Z. Maksymowicz, Physica B 149, 240 (1988).
${ }^{11}$ N. Hamada and H. Miwa, Prog. Theor. Phys. 59, 1045 (1978).

${ }^{12}$ M. E. Elzain and D. E. Ellis, J. Magn. Magn. Mater. 65, 128 (1987).

${ }^{13}$ D. E. Ellis, Int. J. Quantum Chem. 2S, 35 (1968); D. E. Ellis and G. S. Painter, Phys. Rev. B 2, 2887 (1970); G. S. Painter and D. E. Ellis, ibid. 12, 4747 (1970).

${ }^{14}$ U. von Barth and L. Hedin, J. Phys. C 5, 1629 (1972).

${ }^{15}$ C. Umrigar and D. E. Ellis, Phys. Rev. B 21, 2 (1980); D. E. Ellis, G. A. Benesh, and E. Byrom, ibid. 20, 1198 (1979); B. Delley, D. E. Ellis, and A. J. Freeman, J. Magn. Magn. Mater. 30, 71 (1982).

${ }^{16}$ A. Rosen, D. E. Ellis, H. Adachi, and F. W. Averill, J. Chem. Phys. 65, 9, 3629 (1976).

${ }^{17}$ B. Dritler, N. Stefanou, S. Blügel, R. Zeller, and P. H. Dederichs, Phys. Rev. B 40, 8203 (1989).

${ }^{18}$ J. Krause, J. Schaff, C. Paduani, and M. I. da Costa, Jr. (unpublished). 